\title{
Causal trajectories description of atom diffraction by surfaces
}

\author{
A. S. Sanz* and F. Borondo ${ }^{\dagger}$ \\ Departamento de Química, C-IX, Universidad Autónoma de Madrid, Cantoblanco-28049 Madrid, Spain
}

S. Miret-Artés

Instituto de Matemáticas y Física Fundamental, C.S.I.C., Serrano 123, 28006 Madrid, Spain

(Received 29 June 1999; revised manuscript received 15 September 1999)

\begin{abstract}
The method of quantum trajectories proposed by de Broglie and Bohm is applied to the study of atom diffraction by surfaces. As an example, a realistic model for the scattering of $\mathrm{He}$ off corrugated $\mathrm{Cu}$ is considered. In this way, the final angular distribution of trajectories is obtained by box counting, which is in excellent agreement with the results calculated by standard $S$ matrix methods of scattering theory. More interestingly, the accumulation of quantum trajectories at the different diffraction peaks is explained in terms of the corresponding quantum potential. This nonlocal potential "guides" the trajectories causing a transition from a distribution near the surface, which reproduces its shape, to the final diffraction pattern observed in the asymptotic region, far from the diffracting object. These two regimes are homologous to the Fresnel and Fraunhofer regions described in undulatory optics. Finally, the turning points of the quantum trajectories provide a better description of the surface electronic density than the corresponding classical ones, usually employed for this task.
\end{abstract}

\section{INTRODUCTION}

From its beginning quantum mechanics has revealed as a very successful and powerful theory to describe nature. However, the standard formalism in terms of probabilities is often unable to provide a satisfactory intuitive insight into the underlying physical processes, as it is the case for the corresponding classical description. The situation is even worse if one takes into account that there must be some kind of correspondence between the predictions of both mechanics in the appropriate limits (large quantum numbers, $\hbar$ $\rightarrow 0, \ldots .{ }^{1}$

Some alternative formalisms of quantum mechanics have been proposed in the literature. ${ }^{2-5}$ Feynman path integrals provide a statistical approach taking into account all possible path connecting the initial and final points. Madelung derived a "hydrodynamical" formulation of quantum mechanics by introducing a wave function in polar form into the Schrödinger equation. Based on this method and using de Broglie's concept of pilot waves, Bohm developed a formalism in which the initial and final states of a process are connected causally by quantum trajectories.

Recently, there has been a renewed interest from the computational point of view in the de Broglie-Bohm (BB) theory as a tool to study quantum motion. ${ }^{6}$ In some early papers, Hirschfelder et al., ${ }^{7}$ and Dewdney and Hiley ${ }^{8}$ studied the scattering by a square potential barrier, and Philippidis et al. ${ }^{9}$ considered the diffraction through two slits. More recently, Dewdney et al. ${ }^{10}$ explained spin superposition, spin measurements, Einstein-Podolsky-Rosen spin correlations and angular momentum measurements in terms of individual particle trajectories (with continuosusly variable spin vectors), Brown et al. ${ }^{11}$ considered the problem of identical particles within the BB formalism, Oriol et al. applied it to the simulation of resonant tunneling diodes, ${ }^{12}$ and Leavens ${ }^{13}$ and Muga et al. ${ }^{14}$ used it to define arrival times. Chaos has also been studied with Bohmian trajectories, ${ }^{15-19}$ showing examples of exponential divergence. Also, Dey et al. ${ }^{20}$ and Wyatt et $a l_{.}{ }^{21}$ used the quantum fluid dynamical representation to study the photodissociation of $\mathrm{NOCl}$ and $\mathrm{NO}_{2}$, and model collinear chemical reactions, respectively. Finally, other intersting studies of BB quantum trajectories can be found in Ref. 22.

The purpose of this paper is to present a study of quantum effects in atom-surface scattering using the causal BB theory. It is widely accepted that diffraction patterns arising from atom-surface scattering are exclusively the result of the interference of each incident particle with itself, and the broadening of the diffractive peaks a consequence of instrumental imperfections. ${ }^{23}$ Each particle produces its own diffraction probability pattern, and the total one is due to the incoherent superposition of such individual probabilities weighted according to the energy and angular distributions of the incident atomic beam. Thus, the result of an experimental measurement is simply the convolution of the diffraction pattern of individual particles with the instrument response function, so that the latter can be obtained by deconvolution of the former. In this work, we show how this scheme is naturally supported by the theoretical framework given by the BB theory. The quantum potential which appears in this theory provides a different and clearer insight into quantum interference, without the need to abandon the notion of well defined trajectories. The properties of this potential have been discussed in detail in the literature, and can be found in a number of references; see for example Ref. 6. In the BB theory particles are guided by a surrounding wave, solution of the Schrödinger equation, so that the spacetime orbits of an ensemble of particles reproduce the statistical quantum predictions.

As a working example, we have chosen a realistic model system describing the $\mathrm{He}-\mathrm{Cu}$ surface collisions, which have been extensively studied both experimentally and theoreti- 
cally in the past. The scattering of noble gases, in particular $\mathrm{He}$ atoms, is a very powerful technique to characterize surfaces. This nondestructive technique is only sensitive to the outermost atomic layers, thus providing information on the surface corrugation. ${ }^{24}$ Specifically, the elastic scattering of $\mathrm{He}$ atoms off different corrugated $\mathrm{Cu}$ surfaces exhibits a rich variety of interesting phenomena, such as rainbow patterns, selective adsorption resonances, and threshold resonances, which have been observed experimentally. ${ }^{25}$ From the theoretical point of view, those systems have been extensively studied at quantum level, ${ }^{26}$ and classical dynamics have also proven to be adequate to treat this problem. ${ }^{27}$ In particular, we have shown ${ }^{28,29}$ how the onset of classical chaos marks the appearance of (temporary) vibrational trapping of He atoms by the surface. Also, using a pure classical analysis, a new type of scattering singularity, named skipping singularity, was described ${ }^{30}$ providing a classical view of threshold resonances. ${ }^{31}$ However, this approach, even when corrected semiclassically, ${ }^{32}$ does not satisfactorily account, for example, for the conditions of appearance of selective adsorption resonances. ${ }^{33}$

The organization of the paper is as follows. In the next section a brief description of the BB theory and the procedure used for the calculation of the time-dependent wave function needed to compute the quantum potential seen by the trajectories are given. The results obtained are presented and discussed in Sec. III, and finally the conclusions of the present paper are summarized in Sec. IV.

\section{METHOD}

\section{A. The quantum trajectories formalism of de Broglie-Bohm}

Following a suggestion by Madelung, ${ }^{3}$ writing the wave function in polar form

$$
\Psi(\mathbf{r}, t)=R(\mathbf{r}, t) \exp [(i / \hbar) S(\mathbf{r}, t)],
$$

where $R=\Psi^{*} \Psi$ and $S=(\hbar / 2 i)\left(\ln \Psi-\ln \Psi^{*}\right)$ are two real functions of position and time, allows one to recast the timedependent linear Schrödinger equation in the "hydrodynamic" form

$$
\begin{gathered}
\frac{\partial R^{2}}{\partial t}+\nabla\left(\frac{R^{2} \nabla S}{m}\right)=0, \\
\frac{\partial S}{\partial t}+\frac{(\nabla S)^{2}}{2 m}+V+Q=0 .
\end{gathered}
$$

These expressions are the continuity and "quantum" Hamilton-Jacobi equations, respectively. The last term in Eq. (2b) is the quantum potential defined as

$$
Q=-\frac{\hbar^{2}}{2 m} \frac{\nabla^{2} R}{R},
$$

which together with the classical potential $V$ determines the force acting on the system, and then its dynamics. The correspondence principle in this formalism is mathematically expressed as $Q \rightarrow 0$, leading to a continuous transition from quantum to classical mechanics. As can be seen from Eqs. (2), the quantum potential $Q$, which depends on $\mathbf{r}$ and $t$, is determined by the quantum state, directly through $R$ and, due to the coupled partial differential equations, also on $S$. Moreover, $Q$ is singular at the nodes of the wave function causing that the quantum trajectories avoid such regions. Among the most noticeable properties of this potential are that $Q$ is (in general) nonseparable, nonlocal, state or context dependent, and is not mediated by the exchange of particles.

Finally, an important comment concerning the asymptotic regime in the $\mathrm{BB}$ formalism is in order. Usually, the asymptotic region in a scattering problem is defined as the zone where $V$ is negligible and the particle classically follows a free motion. However, in the BB theory forces are also determined by $Q$, and this potential, as will be seen in the next section, extends well inside the classical asymptotic region.

Similarly to the classical case, in this formalism, quantum trajectories associated to a given quantum state can be calculated from Eq. (2b) by integrating the differential equation

$$
\dot{\mathbf{r}} \equiv \frac{d \mathbf{r}}{d t}=\frac{\mathbf{p}(\mathbf{r}, t)}{m},
$$

where the momentum is given in terms of the quantum action $S$ by

$$
\mathbf{p}(\mathbf{r}, t)=\nabla S(\mathbf{r}, t)=\frac{\hbar}{2 i} \frac{\Psi^{*} \nabla \Psi-\Psi \nabla \Psi^{*}}{|\Psi|^{2}} .
$$

According to the probabilistic interpretation these trajectories are the paths along which probability flows. Clearly an explicit expression for the wave function, $\Psi(\mathbf{r}, t)$, is needed in this type of calculations. The quantum state is therefore defined by $\Psi(\mathbf{r}, t)$ and $\mathbf{r}(t)$, which evolve simultaneously in a deterministic way; it is in this sense that the wave "guides" the particles, each of them starting at different positions.

\section{B. Wave-packet propagation}

Let us now describe very briefly the numerical procedure used to propagate the initial wave packet in our problem. To solve the time-dependent Schrödinger equation and obtain the wave function necessary for the calculation of quantum trajectories in the $\mathrm{BB}$ formalism we have chosen the method proposed by Heller. ${ }^{34}$

As it is well known, Gaussian wave packets remain Gaussian in harmonic potentials, and the expectation values of positions and momenta follow the classical equations of motion, according to Ehrenfest's theorem. For nonharmonic, but smooth potentials, wave packets sufficiently narrow in comparison with the spatial variations of the potential, have to be built in order for the propagation be well described by the quadratic approximation. Thus Heller's approach assumes a Gaussian wave function as solution of Schrödinger equation

$$
\Psi(\mathbf{r}, t)=\exp \left[\frac{i}{\hbar}\left(\mathbf{r}-\mathbf{r}_{t}\right) \boldsymbol{A}_{t}\left(\mathbf{r}-\mathbf{r}_{t}\right)+\frac{i}{\hbar} \mathbf{p}_{t}\left(\mathbf{r}-\mathbf{r}_{t}\right)+\frac{i}{\hbar} \gamma_{t}\right],
$$

where the parameters, $\mathbf{r}_{t}$ and $\mathbf{p}_{t}$, defining the center of the Gaussian function, evolve according to the classical equations of motion 


$$
\begin{gathered}
\dot{\mathbf{r}}_{t}=\left(\frac{\partial H}{\partial \mathbf{p}}\right)_{\boldsymbol{p}=\boldsymbol{p}_{t}}, \\
\dot{\mathbf{p}}_{t}=-\left(\frac{\partial H}{\partial \mathbf{r}}\right)_{\boldsymbol{r}=\boldsymbol{r}_{t}},
\end{gathered}
$$

and the remaining complex parameters, $\mathbf{A}_{t}$ matrix, and $\gamma_{t}$, controlling the shape and phase of the Gaussian function respectively, are governed by the time evolution equations

$$
\begin{gathered}
\dot{\mathbf{A}}_{t}=-2 \mathbf{A}_{t} \boldsymbol{\mu}^{-1} \mathbf{A}_{t}-\frac{1}{2} \mathbf{V}^{\prime \prime}\left(\mathbf{r}_{t}\right), \\
\dot{\gamma}_{t}=i \hbar \operatorname{Tr}\left(\boldsymbol{\mu}^{-1} \mathbf{A}_{t}\right) \mathbf{p}_{t} \dot{\mathbf{r}}_{t}-E
\end{gathered}
$$

obtained by introducing Eq. (6) into the time-dependent Schrödinger equation. Here $\boldsymbol{\mu}$ is the mass matrix, "Tr" stands for the trace of the corresponding matrix, and $V^{\prime \prime}$ is the second derivative of the external potential in Cartesian coordinates. Notice, as stated before, that this approximation is only exact for strictly quadratic potentials.

Once the time evolution equations for the Gaussian function parameters are known, the next step consists of performing the simulation of the elastic scattering process. For this goal, calculations are carried out using an initial wave packet placed far enough from the interaction region, which consist of a plane wave with a well defined incident energy

$$
\begin{aligned}
\Psi(\mathbf{r}, t=0)= & \frac{1}{\sqrt{k_{z 0}}} e^{i \mathbf{k}_{0} \mathbf{r}}=C \int \exp \left[\frac{i}{\hbar}\left(\mathbf{r}-\mathbf{r}_{0}\right) \boldsymbol{A}_{0}\left(\mathbf{r}-\mathbf{r}_{0}\right)\right. \\
& \left.+\frac{i}{\hbar} \mathbf{p}_{0}\left(\mathbf{r}-\mathbf{r}_{0}\right)+\frac{i}{\hbar} \mathbf{p}_{0} \mathbf{r}_{0}+\frac{i}{\hbar} \gamma_{0}\right] d \mathbf{r}_{0},
\end{aligned}
$$

where $\mathbf{k}_{0}=\mathbf{p}_{0} / \hbar$ is the incident wave vector. This wave packet is approximated in our case as a sum of Gaussian functions

$$
\begin{aligned}
\Psi(\mathbf{r}, t=0)= & C \sum_{n=1}^{N} \exp \left[\frac{i}{\hbar}\left(\mathbf{r}-\mathbf{r}_{0}^{n}\right) \boldsymbol{A}_{0}^{n}\left(\mathbf{r}-\mathbf{r}_{0}^{n}\right)+\frac{i}{\hbar} \mathbf{p}_{0}^{n}\left(\mathbf{r}-\mathbf{r}_{0}^{n}\right)\right. \\
& \left.+\frac{i}{\hbar} \mathbf{p}_{0}^{n} \mathbf{r}_{0}^{n}+\frac{i}{\hbar} \gamma_{0}^{n}\right],
\end{aligned}
$$

where $N$ is the number of Gaussians used, and the centers of the Gaussians, $\mathbf{r}_{0}^{n}$, are chosen to cover the lenght spanned by a given number of unit cells. Obviously, by increasing the value of $N$ and the number of unit cells taken, a better approximation to the wave function $\Psi$ is obtained.

The initial wave packet is then propagated in time until, after diffraction, it reaches the asymptotic region again. Diffraction intensities are then computed as the square modulus of the $S$-matrix elements obtained from the asymptotic wave function by projection onto final states represented by outgoing plane waves ${ }^{34}$

$$
\begin{aligned}
S_{0}^{g}= & C^{\prime} \sqrt{k_{z}^{g}} \sum_{n=1}^{N}\left(\frac{\pi^{3}}{\operatorname{det} \boldsymbol{A}_{0}^{n}}\right) \exp \left[\frac{-i \hbar}{4}\left(\mathbf{k}_{t}^{n}-\mathbf{k}^{g}\right)\right. \\
& \left.\times\left(\boldsymbol{A}_{0}^{n}\right)^{-1}\left(\mathbf{k}_{t}^{n}-\mathbf{k}^{g}\right)-i \mathbf{k}^{g} \mathbf{r}_{t}^{n}+i \mathbf{k}_{0}^{n} \mathbf{r}_{0}^{n}+\frac{i}{\hbar} \gamma_{t}^{n}\right]
\end{aligned}
$$

where $g$ indicates the diffraction channel satisfying the diffraction condition for the corresponding final state given by

$$
\mathbf{K}_{f}=\mathbf{K}_{i}+\mathbf{G} \text {, }
$$

where $\mathbf{K}_{f}, \mathbf{K}_{i}$ are the parallel components of the final and initial wave vectors $\mathbf{k}_{f}$ and $\mathbf{k}_{i}$, respectively, and $\mathbf{G}$ is the reciprocal lattice vector. This method has been succesfully applied to the study of $\mathrm{He}-\mathrm{LiF}$ surface ${ }^{35}$ and $\mathrm{He}-\mathrm{Cu}$ surface scattering. ${ }^{36}$

\section{Model system}

In this paper we study the scattering of ${ }^{4} \mathrm{He}$ atoms off a $\mathrm{Cu}(110)$ surface which is weakly corrugated. In these conditions the out-of-plane collisions are negligible, and thus a 2D model can be used. The interaction potential is described by a corrugated Morse function

$$
V(x, z)=V_{M}(z)+V_{C}(x, z)
$$

with the Morse function

$$
V_{M}(z)=D\left(1-e^{-\alpha z}\right)^{2}
$$

and the coupling term

$$
V_{C}(x, z)=D e^{-2 \alpha z}\left[0.03 \cos \frac{2 \pi x}{a}+0.0004 \cos \frac{4 \pi x}{a}\right]
$$

Coordinates $z$ and $x$ are defined as perpendicular and parallel to the surface, respectively, and the values for the Morse parameters $\left(\alpha=1.05 \AA^{-1}\right.$ and $\left.D=6.35 \mathrm{meV}\right)$ and for the unit cell length $(a=3.6 \AA)$ for this surface have been taken from the literature. ${ }^{25}$

Since the centers of the Gaussian packets evolve according to classical Hamilton equations of motion [see Eqs. (7a) and (7b)], their initial values are selected according to the following classical relations: ${ }^{29}$

$$
\begin{gathered}
z_{0}=z_{\max }, \\
x_{0}=-z_{\max } \tan \theta_{i}+b a, \\
P_{z 0}=-\sqrt{2 m E} \cos \theta_{i}, \\
P_{x 0}=\sqrt{2 m E} \sin \theta_{i},
\end{gathered}
$$

where $z_{\max }$ represents a value of $z$ sufficiently large so that the classical interaction potential can be neglected, $b$ is the normalized impact parameter $(0 \leqslant b \leqslant 1)$ covering the unit cell length, $E$ the collision energy, and $\theta_{i}$ the initial incident angle. This angle $\theta_{i}$ initially determines the partition of the total energy and momentum between the two modes. 


\section{Initial conditions for the quantum trajectories and box-counting process}

Computation of the BB quantum trajectories implies the integration of Eq. (4) starting at some definite initial positions. Although quantum mechanically all initial points are possible in principle, their probabilities must be distributed accordingly to the particle probability density given by the square modulus of the corresponding initial wave function in order to correctly reproduce the experimental situation under study. In our case this is accomplished by propagating a large ensemble of trajectories all starting at the same value of $z=z_{\max }$, and with a distribution of the parallel position $x$ proportional to $\left|\Psi\left(x, z_{\max }, t=0\right)\right|^{2}$. These trajectories are stopped when observed to follow a straight path for a long enough time. Notice that, as we pointed out in Sec. II A, this happens in general at a distance much larger than that where the classical potential vanishes. In our case, the classical and quantum asymptotic regions start at approximately 12 and $2000 \AA$, respectively. Once this "quantum asymptotical" region has been reached, diffraction intensities are computed by counting the trajectories entering in small boxes of $0.3^{\circ}$ (consistent with the angular resolution of $0.2^{\circ}-0.5^{\circ}$ usually reached by the experimentalists ${ }^{25}$ ) as a function of the final deflection angle. This calculation is to be compared with the quantum mechanical $S$-matrix theory results obtained from Eq. (10).

\section{RESULTS AND DISCUSSION}

\section{A. Numerical details of the wave packet propagation}

When applying Heller's propagation method to atomsurface scattering some technical points have to be carefully considered. ${ }^{35}$ In the first place, the initial wave packet has to be spread over a spatial region large enough to allow a suitable sampling of the surface corrugation details. This is accomplished, as explained in Sec. II D, by using a linear combination of Heller's packets covering a region corresponding to several unit cells. In the second place, the value for the imaginary part of the shape $\mathbf{A}_{0}$ matrix must be chosen as to guarantee the minimum spreading of the whole packet when hitting the surface and the interaction is strong. Taking this into account, 10 Gaussian functions per unit cell were used covering 10 unit cells; so that 100 Gaussian functions were taken in total to simulate a plane wave of $21 \mathrm{meV}$ of energy at normal incidence $\left(p_{x, 0}=0\right)$.

In Fig. 1 we show the evolution of the wave packet, by displaying snapshots of the probability density at three different times: 0, 1.3, and 203 ps. These times have been selected as to show the system at representative moments; namely, at its initial position $(t=0)$ far away from the interaction region, when hitting the surface and the wave packet begins to be dispersed by the interaction $(t=1.3 \mathrm{ps})$, and finally in the asymptotic region at $t=203 \mathrm{ps}$. In this last snapshot, the splitting of the initial wave packet into three components corresponding to the specular and two first order diffraction channels is clearly observed, the intensity of the former being much bigger than that of the other peaks.

\section{B. Quantum trajectories and quantum potential}

To compute the diffraction intensities for our problem we have propagated $\sim 1300$ quantum trajectories, which are
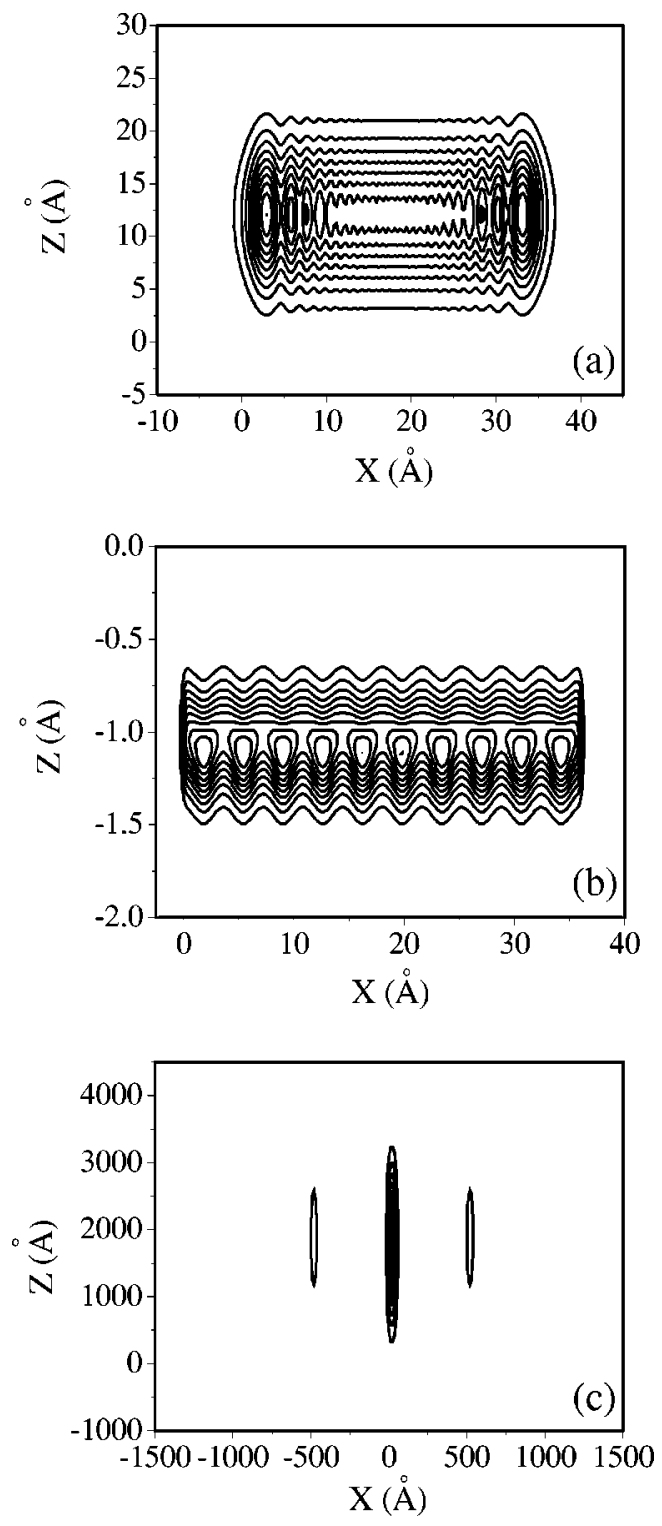

FIG. 1. Snapshots of the wave packet showing the probability density at different points of its evolution: (a) 0 ps (initial wave packet), (b) $1.3 \mathrm{ps}$ (when the packet hit the surface), and (c) $203 \mathrm{ps}$ (when the packet is in the asymptotic region).

shown in Fig. 2. To make the picture clearer we have only represented one tenth of the original trajectories, and we have not plotted their incident parts, where due to the lack of interaction nothing interesting happens. Similarly to what is observed in the last snapshot of Fig. 1(c), it is easily recognized here that the trajectories naturally accumulate along the directions corresponding to the three open diffraction channels at the final angles $0^{\circ}$ and $\pm 15.96^{\circ}$, obtained from the diffraction condition of Eq. (11). Notice that due the large scale used in the figure very few details are appreciable, hence, for example, trajectories appear almost linear and corrugation does not manifest. In order to make a more detailed analysis, we present in Fig. 3 enlargements of three different regions of Fig. 2. In the first panel [Fig. 3(a)] the dynamics in the zone closest to the $\mathrm{Cu}$ surface is shown. To discuss the connection with the surface corrugational features we have also included the equipotential corresponding to $21 \mathrm{meV}$; to make it more obvious we have magnified the 


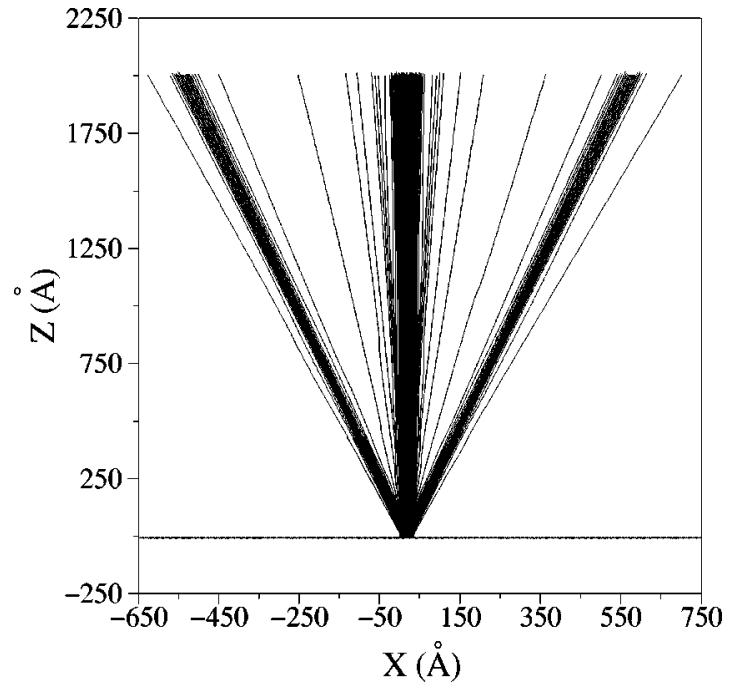

FIG. 2. Quantum trajectories for the scattering of He atoms off a $\mathrm{Cu}(110)$ surface at a total energy of $21 \mathrm{meV}$ and normal incidence. To make the figure more readable only the outgoing part of the trajectories has been displayed. The corresponding classical equipotential contour has also been included; due to the large scale used the effect of the corrugation is not appreciable. Trajectories naturally accumulate along the three open diffraction channels corresponding to orders 0 and \pm 1 , respectively.

corresponding values of $z$ by a factor of 100 . It is seen that the quantum trajectories display undulations along (aproximately) vertical lines, in a quite regular fashion. In this way it appears a first layer of kinks on top of the corrugation function maxima; in a second shell, kinks sit on the minima, and so on, giving rise to a quite regular pattern which constitutes a good image of the diffracting surface. Notice that this pattern formed by the quantum trajectories is due (at least in part) to the fact that they cannot cross each other. ${ }^{6}$

In the second enlargement, Fig. 3(b), a region further apart from the surface is shown. Here the undulatory pattern progressively disappears, and the trajectories becomes more rectilinear, altough still showing noticeable kinks when avoiding crossing. Also the quantum trajectories start to clump along three incipient nonoverlaping groups which are reminiscent of the three open diffraction channels that are obtained for $21 \mathrm{meV}$ and perpendicular incidence. The density of trajectories in each subset is an indication of the diffraction intensity at each of these channels.

Finally, in Fig. 3(c), which corresponds to the zone of very large values of the perpendicular coordinate $z$ (and consequently of $x$ ), trajectories enter the asymptotic region and become clearly rectilinear. At the same time, the three incipient "beams" get completely defined along the diffraction angles.

Cases (a) and (c) depicted in Fig. 3 are analogous to the regions that appear in classical optics diffraction phenomena, where two well defined aproximations, the near field or Fresnel regime and the far field or Fraunhofer regime, exist. The difference between both is that while the former gives spatial information about the diffracting object (its form), the latter gives information about the effects produced by that diffracting object (the redistribution of momenta). We will use this fact for labeling the enlargements by identifying them with their homologies in optics. The limit between
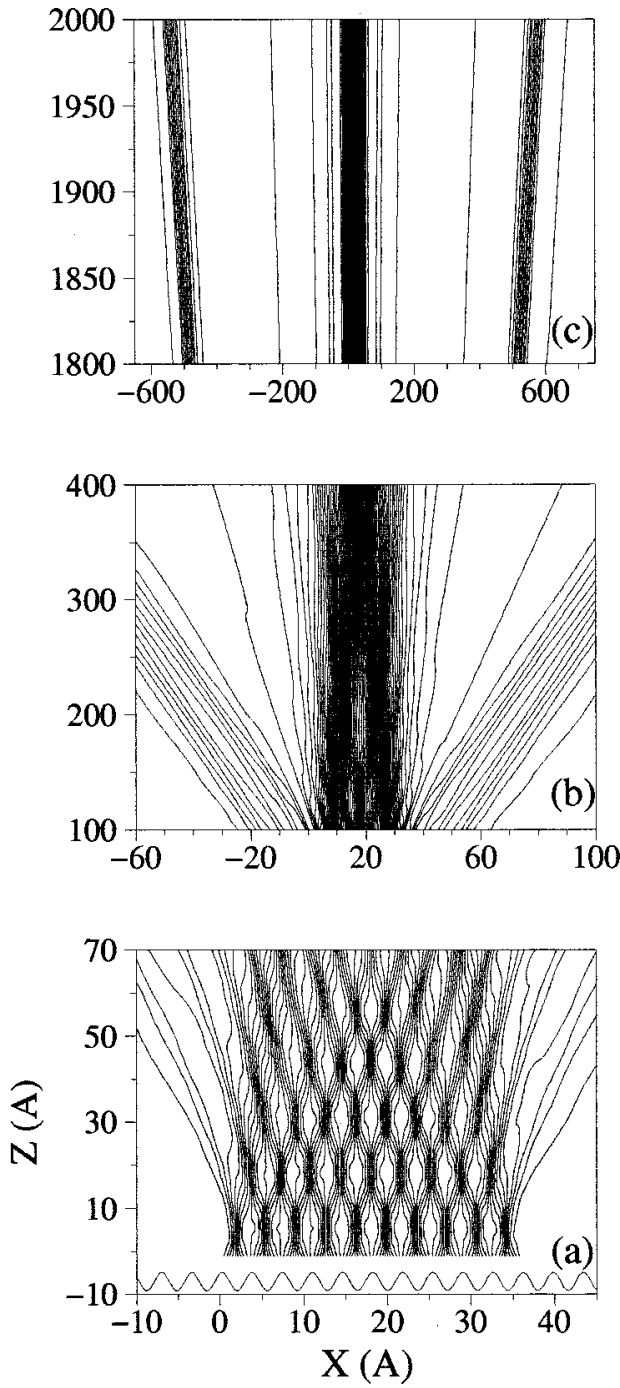

FIG. 3. Different enlargements of Fig. 2 corresponding to: (a) Fresnel, (b) transition, and (c) Fraunhofer regions of the quantum trajectories. In panel (a) the coordinate $z$ of the equipotential has been magnified by a factor of 100 in order to make the corrugation effects obvious.

these two regions is not well defined, since there is a continuous transition from one into the other. However, in classical optics it can be given a typical range as a characteristic length separating these two regimes $\left(r \gg R_{0}\right) .{ }^{37}$ For a general aperture this range is given by

$$
R_{0}=\frac{d^{2}}{4 \pi \lambda},
$$

where $d$ represents the size of the aperture and $\lambda$ is the wavelenght of the incident beam. When this criterion is applied to our case a value of $\sim 126 \AA$, in good agreement with the results of Fig. 3, is obtained.

The behavior that we have just described can be very well understood in terms of an effective potential, obtained by addition of the quantum $Q$ and classical $V$ potentials, which determines the dynamics of the particles. The corresponding potential is shown in Fig. 4 for the three regions: Fresnel, transition, and Fraunhofer, described above. The troughs or canyons exhibited by the effective potential, which are solely due to the quantum interaction, are regions where the trajectories undergo strong forces, accelerating and decelerating 

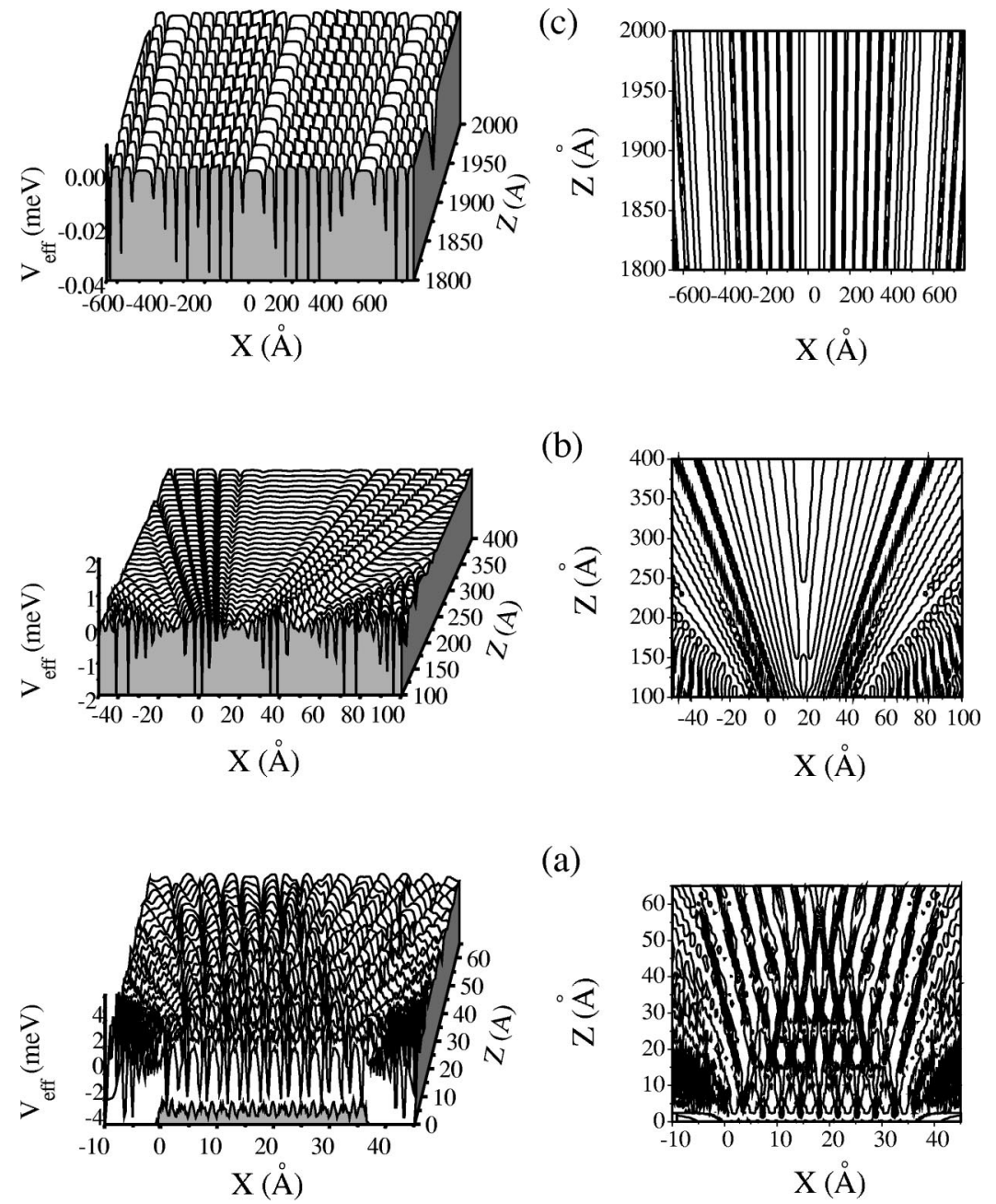

(a)

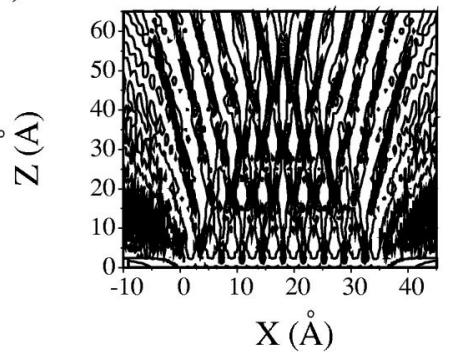

FIG. 4. Effective potential, $V_{\text {eff }}=V+Q$, shown both in $3 D$ and contours plots. Bottom, middle, and top tiers correspond, respectively, to the Fresnel, transition, and Fraunhofer regions. The plateaus and troughs "guiding' the quantum trajectories to the allowed diffraction channels are clearly visible.

the particles which emerge in the plateaus where the quantum force is much weaker and the dynamics smooth. In this way the trajectories are deflected mainly towards the directions that constitute the allowed diffraction channels. Moreover, the strong variations of the effective potential are then responsible for the kinks observed along the trajectories. In comparison to this, the effect of the classical potential is only constrained to a small area in the vicinity of the $\mathrm{Cu}$ surface ( $z<12 \AA$ ), with the effect of dispersing the incident wave packet after the collision.

This type of analysis constitutes a proof of the interpretative power of the $\mathrm{BB}$ theory, which is only possible due to causality. Moreover, the fact that quantum trajectories do not cross in configuration space enables us to know, by following the corresponding "histories," from which part of the initial wave packet each one of them was originated. This is illustrated in Fig. 5. In it, we have represented in the left tier the quantum trajectories of Fig. 2 corresponding to each diffraction channel separately, and in the right part we have indicated, with a thick line, over the probability density profile $\left|\Psi\left(x, z_{\text {max }}, t=0\right)\right|^{2}$ the region where they come from. As can be seen, the specular diffraction channel corresponds to the center of the packet, while channels \pm 1 are originated at the borders. Other more sophisticated dynamical analyses can be performed in the same way; for example one could study the crystalline momentum transfer, or the forces ${ }^{21}$ that act on the different parts of the wave packet.

Another point worth discussing here is that of the surface corrugation. In treatments based on classical trajectories, the turning points give an idea of this corrugation of the diffracting surface. This corrugation is determined by the electronic density at the surface and is well described by the corresponding equipotential line. It is therefore interesting now to consider how this view changes when the quantum trajectories of the $\mathrm{BB}$ theory, determined by the effective potential, are considered. In Fig. 6 (a) the loci formed by the turning points of the quantum (full line) and classical (dashed line) trajectories are presented. As is seen the oscillations in the quantum curve are less pronounced, with the result that the trajectories penetrate less in the classically allowed region and more in the classically forbidden one. In part (b) of the figure the potential profiles: $V$ (dashed line), $Q$ (dotted line), and $V+Q$ (full line), evaluated at the quantum turning points are presented. The most important result is that, as a consequence of the quantum interaction, which is dephased from the classical one, the effective potential is higher by $\sim 7$ $\mathrm{meV}$. The combination of these two effect explains the attenuation of the corrugation discussed above. 

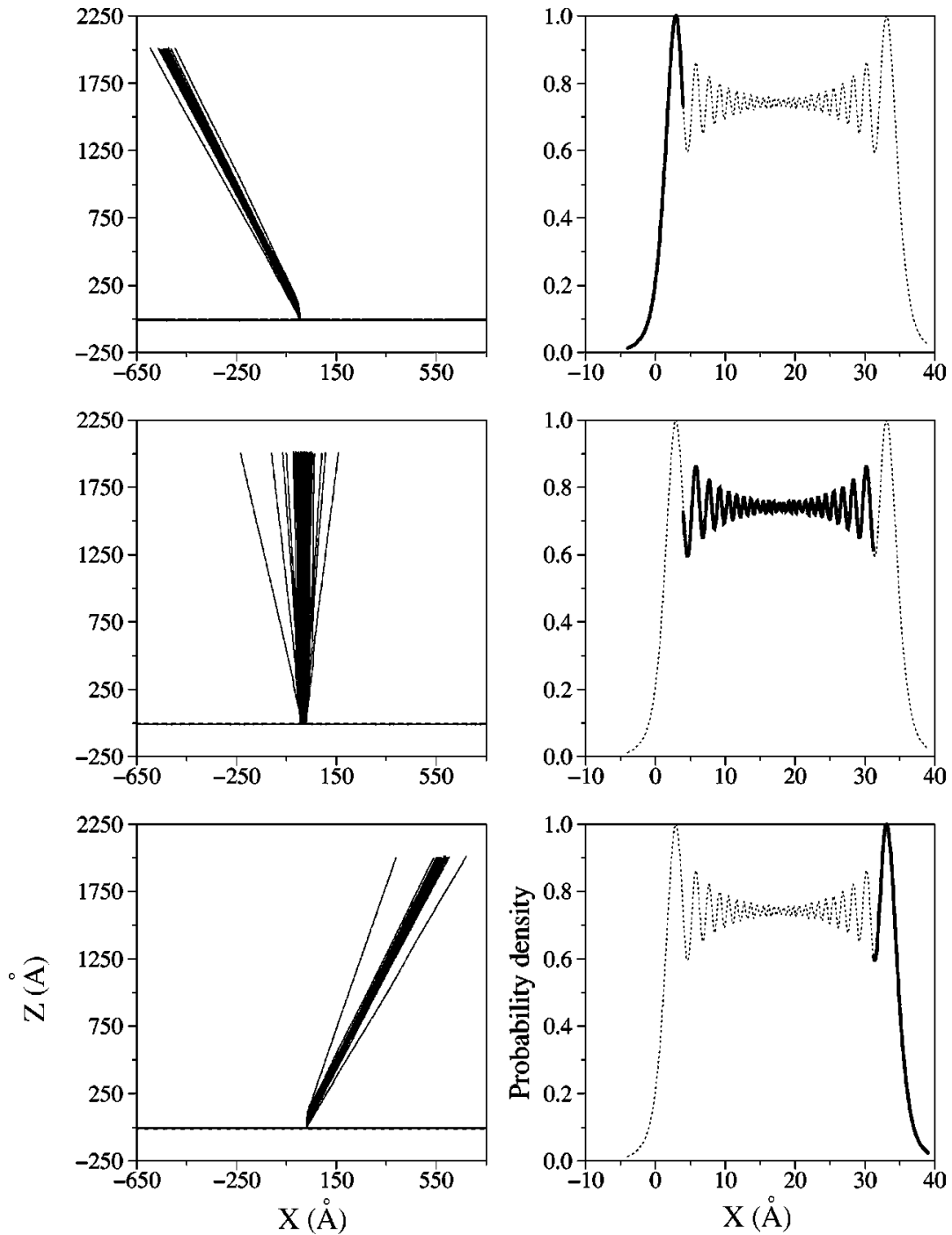

FIG. 5. Connection between quantum trajectories and the initial probability density. In the left tier we present the results of Fig. 2 separated among the three existing diffraction channels, and in the right part the corresponding initial points on the $\left|\Psi\left(x, z_{\max }, t=0\right)\right|^{2}$ probability density profile have been highlighted with thick line.

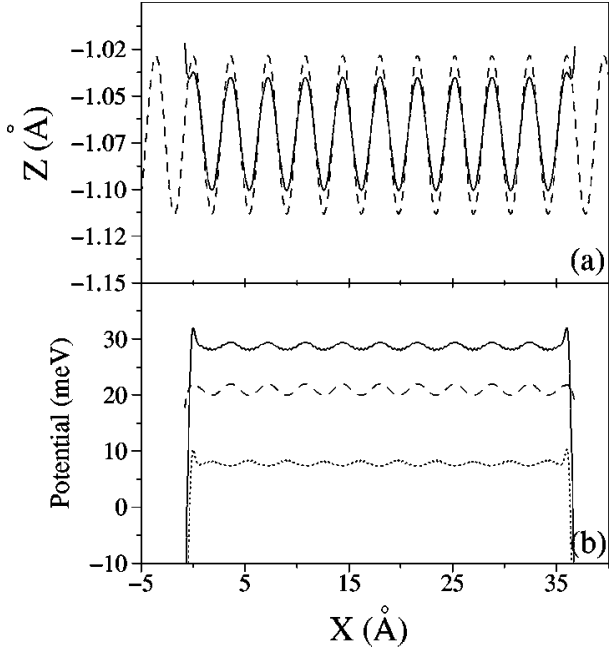

FIG. 6. (a) Loci formed by the turning points of classical (dashed line) and quantum trajectories (full line) of Fig. 2. (b) Quantum (dotted line), classical (dashed line), and effective (full line) potential profiles evaluated at the quantum turning points presented in (a).

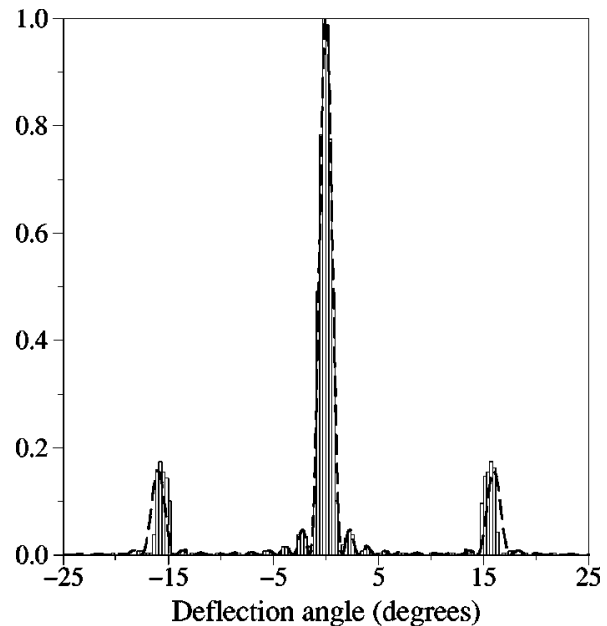

FIG. 7. Diffraction intensities as a function of the deflection angle for the scattering of $\mathrm{He}$ atoms off a $\mathrm{Cu}(110)$ surface at a total energy of $21 \mathrm{meV}$ and normal incidence. The histogram corresponds to the results obtained from the angular distribution of quantum trajectories and the solid line to $S$-matrix calculation. 
Finally, in Fig. 7 the results concerning the angular distribution of the quantum trajectories, computed by the boxcounting technique described in Sec. II D, are shown in the form of a histogram. Three peaks are obtained corresponding to orders 0 (specular) and \pm 1 , centered at $0^{\circ}$ and $\sim \pm 15.7^{\circ}$, being the former the most intense by a factor of 5.8. We also show in this plot the results calculated by the $S$-matrix method as implemented by Drolshagen and Heller. ${ }^{35}$ The agreement is fairly good, and the positions of the peaks coincide very well with the values predicted by the diffraction condition of Eq. (11). However, a small discrepancy between the quantum trajectories and $S$-matrix results is observed in Fig. 7 for the positions of the \pm 1 peaks. This can be attributed to the approximation used in the wave packet propagation, since in Heller's method a quadratic approximation for the potential is assumed.

\section{CONCLUSION}

Interference and diffraction phenomena are central to any discussion about the interpretation of quantum mechanics. In particular, the study of the double slit experiment in the BB in 1979, where quantum trajectories were explicitly calculated, represented a point of renewed interest in this type of questions. The interpretation in this formalism relies on the fact that particles follow spacetime tracks different from that of a classical particle with the same physical characteristics and moving in the same experimental setup. Furthermore, along the propagation from the source to the screen, particles are surrounded and guided by the wave function. When many particles (or trajectories) are considered, the observed diffraction pattern can be viewed as the result of the selfinterference of all incident particles; in other words, the aspect of the pattern is granular and built up from a series of single-particle events. In the two slits experiment, quantum trajectories split into two subsets, each one coming from a different slit, without any crossings between them.

In this work we have studied a more complicated problem, which is analogous to the optical diffraction by a grating, coming from surface physics: the elastic scattering of $\mathrm{He}$ atoms off corrugated $\mathrm{Cu}$ surfaces but in presence of a periodic, soft potential. This gave us the opportunity to revisit the concepts of near field and far field of optics establishing an homology with quantum mechanics, in the sense that the wave function evolves as an optical wave showing two well differentiated regimes analogous to the Fresnel and Fraunhofer regions appearing in optics. This later regime is more conditioned by the quantum potential than by the classical one, since the former acts in zones where the influence of the surface is negligible, as discussed in Sec. II A. In this sense, the asymptotic region in the theoretical framework given by the BB theory is very different from that in standard quantum or classical mechanics.

Experimentally, diffraction patterns are granular because the detector signal consists of a given number of counts (of $\mathrm{He}$ atoms in this case) per unit time. This is consistent with the concept of quantum trajectories, that as we stated before are the paths along which probability flows, thus connecting causally particles with the counts in the detector. The bright and dark fringes, or variations in intensity in a diffraction pattern has a causal agent, the quantum potential. Even more, from diffraction intensities it is possible, in principle, to infer which trajectories contribute to each maximum.

This formalism can be related to the path integral approach, since each of these spacetime tracks followed by particles can be viewed as a "superposition" of classical paths, taking into account their interference. ${ }^{6}$ However, there is an important difference between these two approaches. For any given initial condition, there exists only one quantum trajectory taking the particle to the final state, while there are infinitely many Feynman paths connecting them.

To summarize, in this paper a theoretical study of the elastic scattering of $\mathrm{He}$ atoms off a $\mathrm{Cu}(110)$ surface, described by a reallistic 2D interaction potential, has been presented. The collision dynamics have been described using the causal formalism due to Bohm, where particles follow well defined quantum trajectories, which allows to connect the initial and final states. These trajectories are (deterministically) governed by a "quantum" Hamilton-Jacobi equation. Here the concept of wave acquires a new physical meaning, since it gives the particle probability density (in a statistical sense), and at the same time "guides" the trajectory described by the particle. This action takes place only by means of a new additive term in the potential, which is of pure quantum nature since it depends only on the amplitude of the wave function. From the trajectories propagated by this method we have computed, using a box-counting procedure, the final angular distribution of intensities for an energy of $21 \mathrm{meV}$ and normal incidence. The corresponding results are in very good agreement with the values calculated by the standard $S$-matrix method.

Another important result of our paper is the possibility to calculate, using the turning points of quantum trajectories, the electronic density at the surface, a central issue in surface science. This alternative method provides a better description of the surface corrugation that the usual one, based on classical turning points.

In our opinion, the use of the BB theory for the study of processes similar to the one we have studied is in general very powerful, since it provides a causal intuitive interpretation of the underlying dynamics. For example, we are currently applying this method to more complicated scattering situations, in which resonant or rainbow phenomena takes place, in order to gain a deeper understanding of these processes. In particular, the diffraction of He from a single adsorbate or from an island, for which many theoretical and experimental results can be found in the literature, could supply an alternative deeper and more quantitative view of the quantum interference.

\section{ACKNOWLEDGMENTS}

This work was supported in part by DGES (Spain) under Contract Nos. PB95-71, PB95-425, and PB96-76. A.S.S. gratefully acknowledges financial support from the Universidad Autónoma de Madrid. 
*Electronic address: angel.sanz@uam.es

†Electronic address: f.borondo@uam.es

†Electronic address: salvador@fam88.imaff.csic.es

${ }^{1}$ R. L. Liboff, Phys. Today 37, 50 (1984).

${ }^{2}$ R. P. Feynman, Rev. Mod. Phys. 20, 367 (1948); R. P. Feynman and A. R. Hibbs, Quantum Mechanics and Path Integrals (McGraw-Hill, New York, 1965).

${ }^{3}$ E. Madelung, Z. Phys. 40, 332 (1926).

${ }^{4}$ L. de Broglie, Compt. Rend. Acad. Sci. 183, 447 (1926).

${ }^{5}$ D. Bohm, Phys. Rev. 85, 166 (1952); 85, 194 (1952).

${ }^{6}$ P. R. Holland, The Quantum Theory of Motion (Cambridge University Press, Cambridge, 1993); D. Bohm and B. J. Hiley, The Undivided Universe (Routledge, New York, 1993).

${ }^{7}$ J. O. Hirschfelder, A. C. Christoph, and W. E. Palke, J. Chem. Phys. 61, 5435 (1975).

${ }^{8}$ C. Dewdney and B. J. Hiley, Found. Phys. 12, 27 (1982).

${ }^{9}$ C. Philippidis, C. Dewdney, and B. J. Hiley, Nuovo Cimento Soc. Ital. Fis., B 52, 15 (1979); C. Philippidis, D. Bohm, and R. D. Kaye, ibid. 71, 75 (1982).

${ }^{10}$ C. Dewdney, P. R. Holland, A. Kyprianidis, and J. P. Vigier, Nature (London) 336, 536 (1988); C. Dewdney and Z. Malik, Phys. Rev. A 48, 3513 (1993).

${ }^{11}$ H. R. Brown, E. Sjöqvist, and G. Bacciagaluppi, Phys. Lett. A 251, 229 (1999).

${ }^{12}$ X. Oriols, F. Martín, and J. Suñé, Phys. Rev. A 54, 2594 (1996); X. Oriol, J. J. García-García, F. Martín, J. Suñé, T. González, J. Mateos, and D. Pardo, Phys. Status Solidi B 204, 404 (1997); Appl. Phys. Lett. 72, 806 (1998).

${ }^{13}$ C. R. Leavens, Phys. Rev. A 58, 840 (1998).

${ }^{14}$ J. G. Muga, R. Sala, and R. F. Snider, Phys. Scr. 47, 732 (1993); J. P. Palao, J. G. Muga, and C. R. Leavens, Phys. Lett. A 253, 21 (1999).

${ }^{15}$ U. Schwengelbeck and F. H. M. Faisal, Phys. Lett. A 199, 281 (1995); F. H. M. Faisal and U. Schwengelbeck, ibid. 207, 31 (1995).

${ }^{16}$ R. H. Parmenter and R. W. Valentine, Phys. Lett. A 201, 1 (1995); 227, 5 (1997).

${ }^{17}$ G. Iacomelli and M. Pettini, Phys. Lett. A 212, 29 (1996).
${ }^{18}$ S. Sengupta and P. K. Chattaraj, Phys. Lett. A 215, 119 (1996).

${ }^{19}$ S. Konkel and A. J. Makowski, Phys. Lett. A 238, 95 (1998).

${ }^{20}$ B. K. Dey, A. Askar, and H. Rabitz, J. Chem. Phys. 109, 8770 (1998).

${ }^{21}$ C. L. Lopreore and R. E. Wyatt, Phys. Rev. Lett. 82, 5190 (1999); R. E. Wyatt, J. Chem. Phys. 111, 4442 (1999).

${ }^{22}$ H. M. Wiseman, Phys. Rev. A 58, 1740 (1998); A. J. Makowski and S. Konkel, ibid. 58, 4975 (1998).

${ }^{23}$ G. Comsa, in Dynamics of Gas-Surface Interactions, edited by G. Benedek and U. Valbusa, Vol. 21 of Springer Series in Chemical Physics (Springer-Verlag, Berlin, 1982), p. 79.

${ }^{24}$ E. Hulpke, Helium Atom Scattering from Surfaces (SpringerVerlag, Berlin, 1992).

${ }^{25}$ D. Gorse, B. Salanon, F. Fabre, A. Kara, J. Perreau, G. Armand, and J. Lapujoulade, Surf. Sci. 147, 611 (1984); D. Farías and K. H. Rieder, Rep. Prog. Phys. 61, 1575 (1998).

${ }^{26}$ M. Hernández, S. Miret-Artés, P. Villarreal, and G. DelgadoBarrio, Surf. Sci. 274, 21 (1992); S. Miret-Artés, ibid. 339, 205 (1995).

${ }^{27}$ See, for example, R. B. Gerber, Chem. Rev. 87, 92 (1987).

${ }^{28}$ R. Guantes, F. Borondo, C. Jaffé, and S. Miret-Artés, Int. J. Quantum Chem. 52, 515 (1994).

${ }^{29}$ R. Guantes, F. Borondo, and S. Miret-Artés, Phys. Rev. E 56, 378 (1997).

${ }^{30}$ S. Miret-Artés, J. Margalef-Roig, R. Guantes, F. Borondo, and C. Jaffé, Phys. Rev. B 54, 10397 (1996).

${ }^{31}$ R. Guantes, F. Borondo, S. Miret-Artés, J. Margalef-Roig, and J. R. Manson, Surf. Sci. 375, L379 (1997).

${ }^{32}$ R. Guantes, F. Borondo, C. Jaffé, and S. Miret-Artés, Phys. Rev. B 53, 14117 (1996); Surf. Sci. 338, L863 (1995).

${ }^{33}$ F. Borondo, C. Jaffé, and S. Miret-Artés, Surf. Sci. 317, 211 (1994).

${ }^{34}$ E. J. Heller, J. Chem. Phys. 62, 1544 (1975).

${ }^{35}$ G. Drolshagen and E. J. Heller, J. Chem. Phys. 79, 2072 (1983).

${ }^{36}$ M. Hernández, J. Campos-Martinez, S. Miret-Artés, and R. D. Coalson, Phys. Rev. B 49, 8300 (1994).

${ }^{37}$ F. G. Smith and J. H. Thomson, Optics (Wiley, New York, 1989); R. Guenther, Modern Optics (Wiley, New York, 1990). 